\title{
Statement of Capabilities: Micropower Impluse Radar (MIR) Technology Applied to Mine Detection and Imaging
}

\author{
S. G. Azevedo \\ D. T. Gavel \\ J. E. Mast \\ J. P. Warhus
}

March 13, 1995

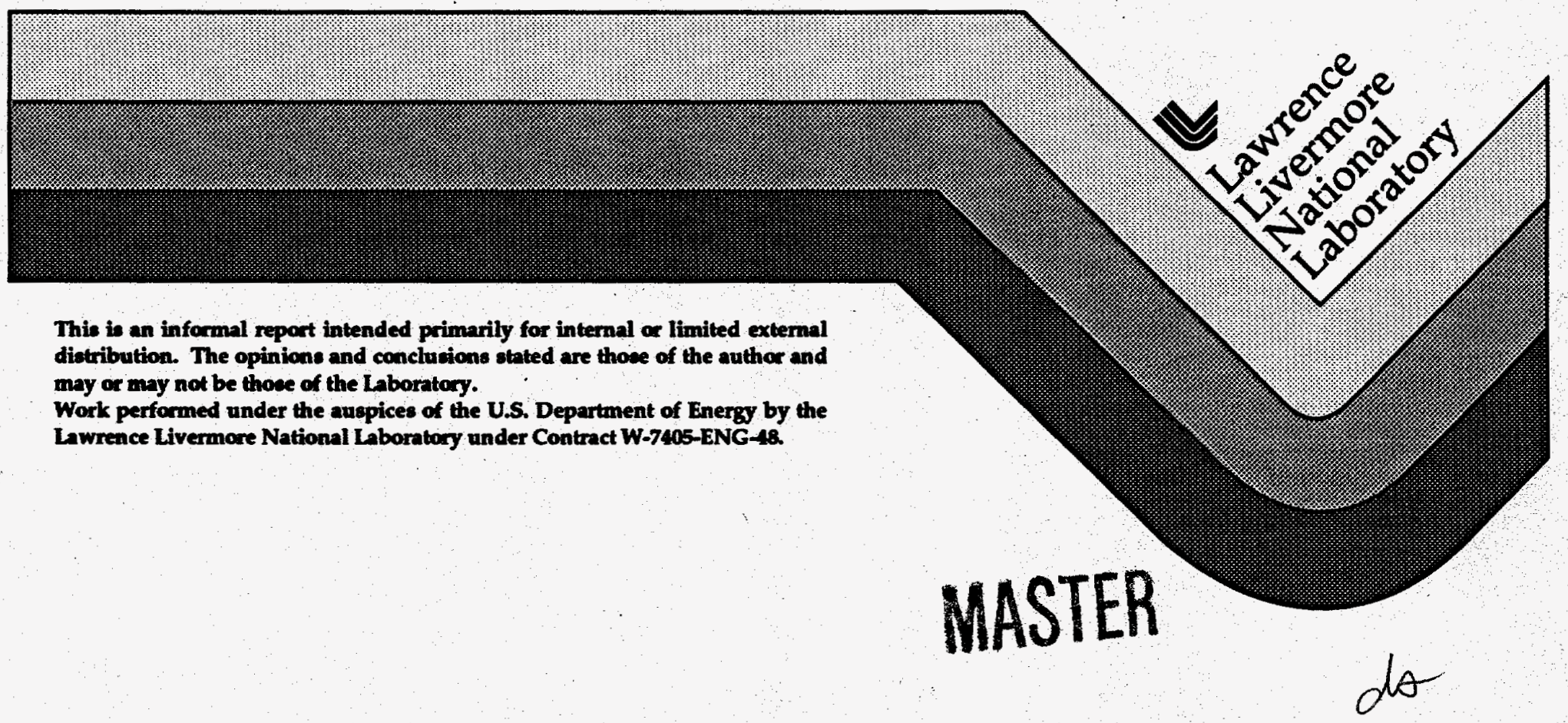




\section{DISCLAMER}

This document was prepared as an account of work sponsored by an agency of the United States Government. Neither the United States Covernment nor the University of California nor any of their employees, makes any warranty, express or implied, or assumes any legal liability or responsibility for the accuracy, completeness, or usefulness of any information, apparatus, product, or process disclosed, or represents that its use would not infringe privately owned rights. Reference herein to any specific commercial product, process, or service by trade name, trademark, manufacturer, or otherwise, does not necessarily constitute or imply its endorsement, recommendation, or favoring by the United States Government or the University of California. The views and opinions of authors expressed herein do not necessarily state or reflect those of the United States Covernment or the University of California, and shall not be used for advertising or product endorsement purposes.

This report has been reproduced directly from the best available copy.

Available to DOE and DOE contractors from the Office of Scientific and Technical Information P.O. Box 62, Oak Ridge, TN 37831

Prices available from (615) 576-8401, FTS 626-8401

Available to the public from the National Technical Information Service

U.S. Department of Commerce 5285 Port Royal Rd.

Springfield, VA 22161 


\title{
Statement of Capabilities: Micropower Impulse Radar (MIR) Technology Applied to Mine Detection and Imaging
}

\author{
S.G. Azevedo, D.T. Gavel, J.E. Mast, and J.P. Warhus \\ Lawrence Livermore National Laboratory \\ Imaging and Detection Program \\ P.O. Box 808 , L-495 \\ Livermore, California 94551
}

\begin{abstract}
The Lawrence Livermore National Laboratory (LLNL) has developed radar and imaging technologies with potential applications in mine detection by the armed forces and other agencies involved in demining efforts. These new technologies use a patented ultra-wideband (impulse) radar technology that is compact, low-cost, and low power. Designated as Micropower Impulse Radar, these compact, self-contained radars can easily be assembled into arrays to form complete ground penetrating radar imaging systems. LLNL has also developed tomographic reconstruction and signal processing software capable of producing high-resolution 2-D and 3-D images of objects buried in materials like soil or concrete from radar data. Preliminary test results have shown that a radar imaging system using these technologies has the ability to image both metallic and plastic land mine surrogate targets buried in 5 to $10 \mathrm{~cm}$ of moist soil. In dry soil, the system can detect buried objects to a depth of $30 \mathrm{~cm}$ and more. This report describes LLNL's unique capabilities and technologies that can be applied to the demining problem. Work described here was performed under the auspices of the U.S. Department of Energy by Lawrence Livermore National Laboratory under Contract No. W-7405-ENG-48.
\end{abstract}

\subsection{Introduction}

1.1 A new radar technology, Micropower Impulse Radar (MIR), has recently been tested to evaluate its viability as a mine detection sensor. These tests show that MIR reliably detects both plastic and metallic land mines and mine surrogates buried in both moist and dry soils. The MIR sensor technology provides several advantages over existing GPR systems including: low cost, low power, lightweight and compact size, and the ability to assemble into compact arrays. Coupled with LLNL's 2-D and 3-D imaging algorithms, MIR offers the potential for a low cost, high performance mine detector that will enhance the reliability and performance of multisensor mine detection systems. This paper describes the new sensor technology and presents examples of its capabilities. In addition, it discusses results from the recent field tests and describes system concepts for vehicle mounted and hand-held mine detectors.

1.2 Previous experience in high-power radar mine detection at $L L N L$ has aided the low-power MIR research. Prior to the MIR development, LLNL investigated and developed a ground penetrating radar on an airborne platform [1] for land mine and unexploded ordnance detection. That system used a $3 \mathrm{kV}$ pulsed transmitter operating in a side-looking mode, standing off about 9 meters from the target area and radiating $1 \mathrm{~ns}$ wide impulses. The radar used a single transmitter/receiver antenna pair to synthesize a 1-D antenna aperture by moving the antennas along the target area. This system successfully detected metallic land mines and land mine surrogates buried in a minefield located at the Nevada Test Site. This system also detected plastic mines, but with significantly lower detection reliability. Mine detection capability was enhanced by 2 -D tomographic imaging algorithms, also developed at LLNL, that were used to reconstruct images of the minefield. We use many of these same components in the current MIR work. 
2.1 The Micropower Impulse Radar (MIR) is a new class of radar because of its (1) low cost and small size, (2) low power requirements, and (3) ultra-wideband (impulse) frequency range. It has the ability to penetrate dielectric materials like wood, concrete, and soil and provide high resolution detection of changes in embedded material dielectric and conductivity properties. A single MIR module is a compact, light-weight, self-contained impulse radar transceiver constructed using low-cost, off-the-shelf components. The low level of radiated RF power $(<100 \mathrm{~mW}$ peak and $<10 \mu \mathrm{W}$ average) and wide bandwidth of the MIR module radiation ensure operator and equipment safety with respect to both RF exposure and ordnance safety concerns. A printed circuit board for a radar module based on MIR technology and designed for use in alarm systems is shown in Figure 1. The imaging MIR module we use for mine detection is of similar size, and its specifications are given in Table 1 .

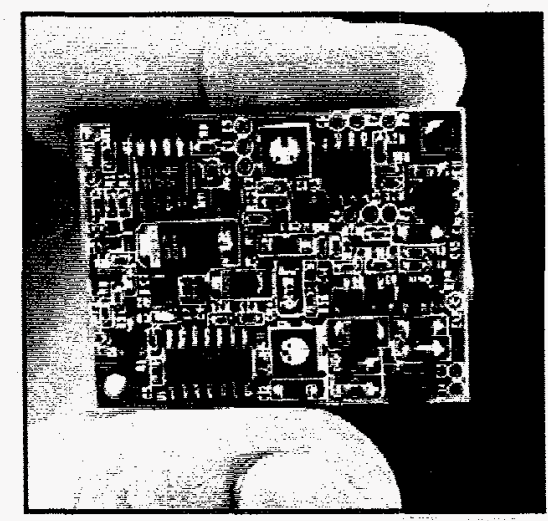

Figure 1. Printed circuit board for a Micropower Impulse Radar motion sensor module.

Table 1. Micropower Impulse Radar imaging module characteristics.

\begin{tabular}{|l|l|}
\hline General & Monostatic \\
\hline Module Configuration & $5 \mathrm{~cm}$ (height), $10 \mathrm{~cm}$ (width), $10 \mathrm{~cm}$ (depth) \\
\hline Overall Dimensions & $<0.23 \mathrm{~kg}$ \\
\hline Weight & 6 to 12 volts, $8 \mathrm{~mA}$ \\
\hline Input Power & $\begin{array}{l}\text { Up to } 10 \mathrm{~m} \text { in air; } 0.2 \text { to } 0.5 \mathrm{~m} \text { in soils } \\
\text { (depends on soil conductivity and standoff range) }\end{array}$ \\
\hline Range (Maximum) & Ultra-wide-bandwidth, impulse waveform \\
\hline Transmitter & $2 \mathrm{MHz}$ \\
\hline Type & $\sim 100 \mathrm{psec}$ \\
\hline Pulse Repetition Frequency & $3.2 \mathrm{GHz}$ (from 0.8 to $4.0 \mathrm{GHz})$ \\
\hline Pulse Rise-time (Radiated) & $<100 \mathrm{~mW}$, peak; $<10 \mu \mathrm{W}$, average \\
\hline Bandwidth & Cavity-backed, resistively-loaded monopole \\
\hline Radiated Power (ERP) & $120^{\circ}$ \\
\hline Antenna: & \\
\hline Beamwidth (-3 dB) & \\
\hline Receiver & Equivalent-time sampler with sensitivity-time control \\
\hline Front End: & $<4.0 \mathrm{GHz}$ \\
\hline Type & $25 \mathrm{~dB}$ \\
\hline Bandwidth & Cavity-backed, resistively-loaded monopole \\
\hline Noise Figure & $120^{\circ}$ \\
\hline Antenna: & \\
\hline Beamwidth (-3 dB) & \\
\hline
\end{tabular}




\section{DISCLAIMER}

Portions of this document may be illegible in electronic image products. Images are produced from the best available original document. 
2.2 The MIR was developed at LLNL in 1993 as an evolution of an R\&D 100 Award Winning Single-shot Transient Digitizer that was designed to record very high-speed transientevents generated by the 100-trillion wattNovapulsed laser. By applying LLNL's historical expertise in data acquisition systems for short-lived laser and nuclear events, laboratory researchers conceived a revolutionary new pulsed radar that is remarkably inexpensive and works well at short ranges (from a few inches to more than 200 feet in air). This novel radar technology has enabled many new applications that would not be practical if they depended upon conventional radar technology. A recent cover story of Popular Science (March 1995) focuses on the MIR and describes many of these applications. More information about the MIR and its development is given in Appendix A.

2.3 For mine detection and imaging, high resolution in both range and cross-range is required. High range resolution is achieved by the ultra-wide bandwidth of the MIR module. The short pulse rise-time of $100 \mathrm{ps}$ yields range resolution of about $1 \mathrm{~cm}$ in typical soils. To achieve high cross-range resolution, an array of MIR elements can be assembled to form a large aperture. The miniature properties of the MIR module make it especially attractive to use in radar arrays. Alternatively, a single radar element collecting samples at evenly-spaced intervals along a line can be used to form a synthetic 1-D array. The resolution achieved depends on the element spacing and the overall dimension of the aperture. For example, we have used a one-meter aperture with one-centimeter spacing (100 synthetic array elements) to achieve cross range resolution of a few centimeters at ten-centimeters depth in soil. To achieve high resolution in two cross-range directions, it is necessary to form a 2-D array on the surface. This generates a full three- dimensional representation of a volume of ground, and can be accomplished by forming a synthetic array in both dimensions.

2.4 LLNL has developed unique imaging software for MIR arrays to provide for high-resolution imaging of buried objects [2]. The imaging software was developed for radar inspection of steel-reinforced concrete bridge decks. This new software does not require direct contact between the sensors and the bridge to obtain inspection data. As a result, the system can collect data over large areas very rapidly. Images reconstructed from the data will enable bridge engineers and inspectors to visualize structural details that were previously only observable using destructive techniques like coring. An example of this imaging capability is shown in Figures 2 and 3 which are, respectively, a photograph of the internal elements of a one-foot-thick concrete slab before concrete was

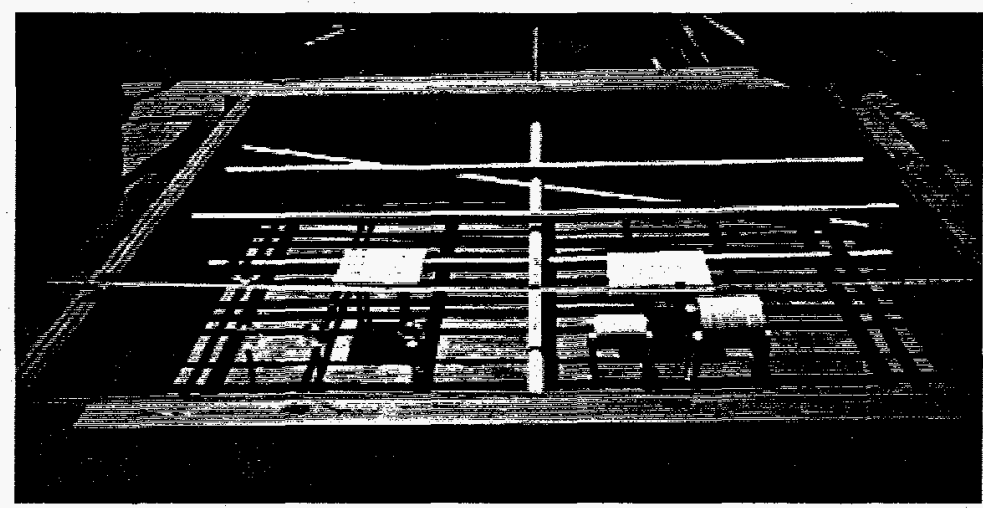

Figure 2. Internal elements of concrete slab before concrete pour.

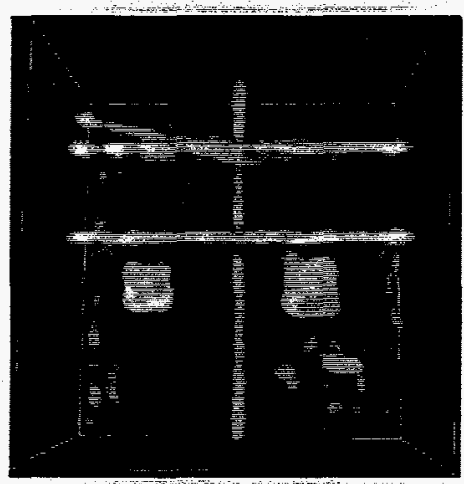

Figure 3. Reconstructed 3-D radar image of elements embedded in the concrete slab (after pour). 
poured and a 3-D image reconstructed from data collected using a single MIR module to form a 2-D synthetic aperture over the poured slab. The radar imaging technology and system concepts developed for bridge deck inspection are directly applicable to the mine detection problem and to other underground imaging problems.

\subsection{MIR Mine Detection Feasibility Tests}

3.1 To evaluate the feasibility of using MIR as a mine detection sensor, we assembled a prototype MIR module into a test system that could be used to make measurements in the field. A single MIR module (with one transmitter and one receiver element) was used for collecting radar data, along synthetic 1-D and 2-D arrays in "look-down" operating mode. It was set up to move along a wooden frame with an air-gap over the ground to simulate a vehiclemounted or hand-held array. To acquire 3-D data, the frame was moved in calibrated steps after each sensor pass. A lap-top computer with data acquisition hardware and software collected the radar data. Figure 4 is a photograph of the system collecting field data. The standoff height for the radar in look down mode varied from 3 to $30 \mathrm{~cm}$.

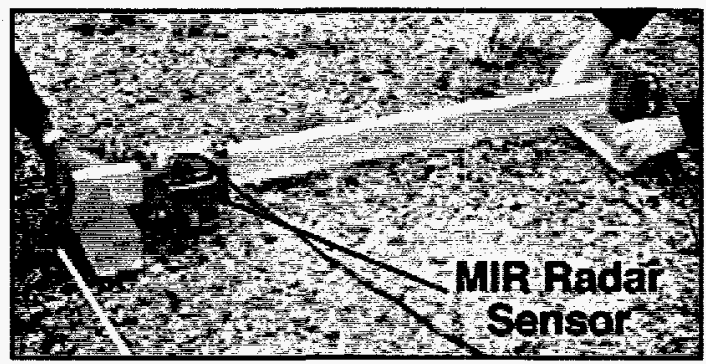

Figure 4. Test system collecting data from a minefield at the Nevada Test Site.

3.2 The field tests were conducted at the Buried Object and Mine Detection Facility at the Nevada Test Site (NTS). The NTS facility appears in the foreground of the photograph in Figure 5. Real anti-tank and anti-vehicular mines (without detonators) and mine surrogates, both plastic and metallic, have been buried in known locations at this facility. Figure 6 shows typical placements of M-19 and VS-2.2 mines before being covered with soil. Natural vegetation, rocks and rubble, and animal burrows were left intact when the mines were buried to maintain realistic conditions. The soil in the test area is made up of alluvium, consisting of Paleozoic fragments and tuff. Soil conductivity is in the range of 5 to 8 millisiemen. The soil was moist, due to rain from the day before, with a dry topmost layer of a few millimeters.

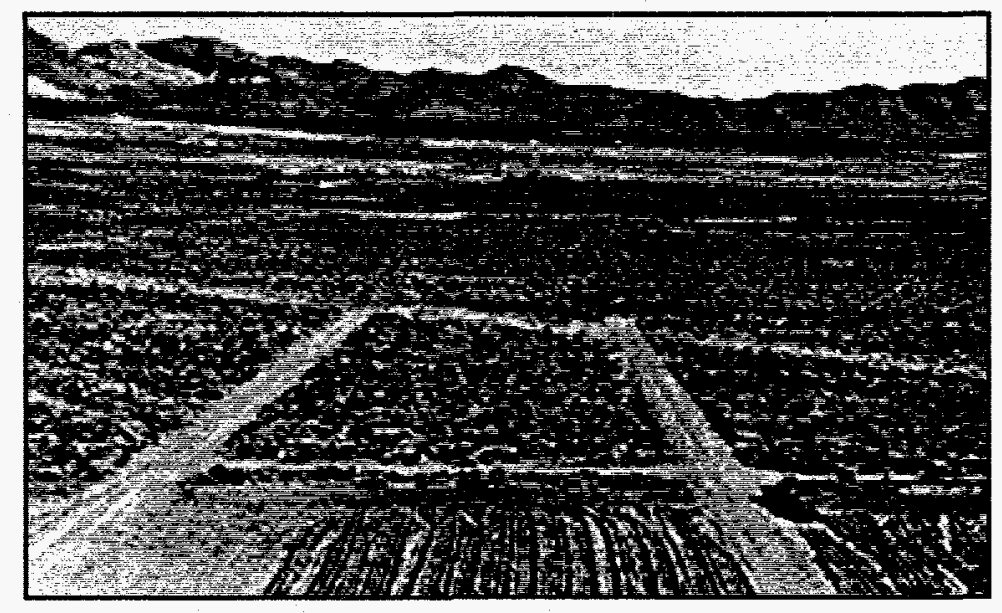

Figure 5. Buried Object and Mine Detection Facility (foreground) at the Nevada Test Site. 


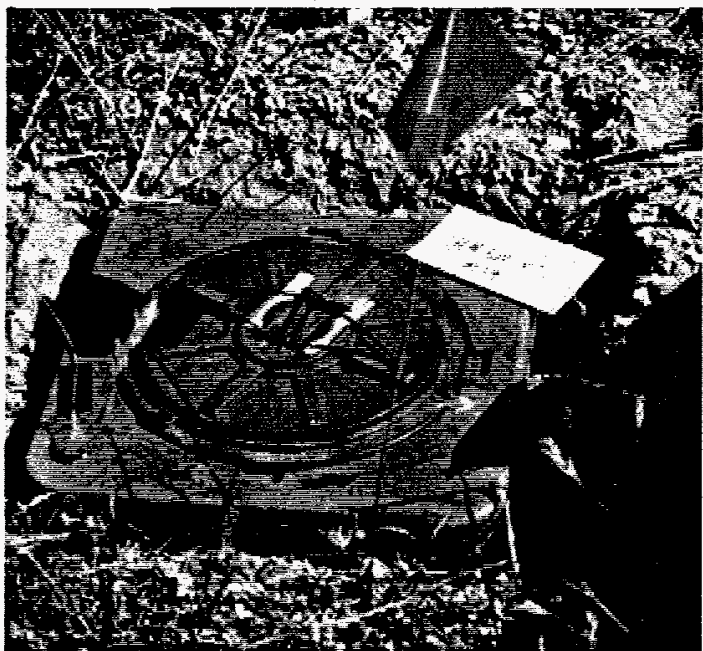

M-19

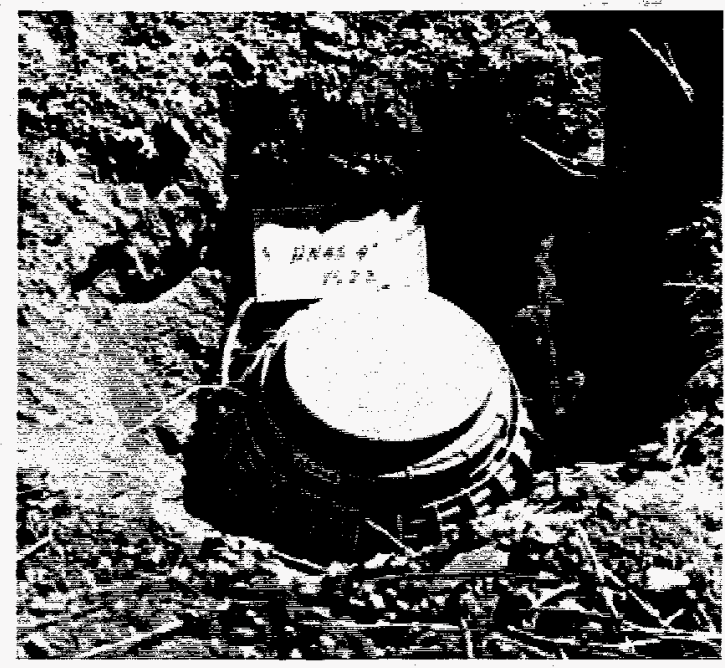

VS-2.2

Figure 6. Typical plastic mine placements before burial at NTS.

3.3 The MIR sensor was scanned over a known M-19 mine location to obtain the 2-D radar return data shown in Figure 7a. This is the traditional B-scan or "waterfall" type display common in radar and ultrasonic imaging. The vertical axis is the time of return, which is roughly proportional to distance from the surface. The horizontal axis is distance along the ground. An alternative 2-D display of the ground cross-section is the reconstructed image in Figure 7b, which shows depth on the vertical axis with the ground surface at the top. Knowledge of the media (air/ground), the scan timing, and the scan geometry are needed to compute this image using diffraction tomography methods. A 486lap-top computerreconstructs the image in less than ten seconds. Notice that the reconstruction gives a clearer delineation of the buried object than the B-scan. Clutter and spurious returns are also visible in the 2-D vertical slice due to buried rocks and other debris, but the dielectric difference between the plastic mine and soil is enough for reliable detection of the mine. Results of the tests show conclusively that real metallic and plastic mines buried roughly $5 \mathrm{~cm}$ beneath the surface can be readily detected and imaged by the MIR system. Metal M-15 mines show a strong reflection from the top of the mine. Plastic M-19 and VS-2.2 mines show reflections from both top and bottom mine surfaces and a characteristic resonance behavior.

3.4 While the 2-D image in Figure $7 \mathrm{~b}$ detects a buried object, full 3-D imaging may be necessary to discriminate between a mine and other object types. A complete grid of radar data was acquired for the two mines in Figure 6 and this time reconstructed at all slices to give a full 3-D volume of imaging elements (voxels). The image reconstruction is accomplished by processing the data collected from a 2-D synthetic array. Now we can display horizontal slices at various depths (Figures 8 and 9 ) to image the object as though peering down at it. Figure 8 shows a sequence of 2-D planar slices of M19 plastic anti-tank mine buried at about a $5.5 \mathrm{~cm} \mathrm{depth}$. Note that the square shape of the mine, its depth in the soil, and its vertical extent, are all clearly evident in the image sequence. Figure 9 shows a similarly reconstructed image of a VS-2.2 plastic anti-tank mine. Its circular cross-sectional shape is also evident in this sequence.

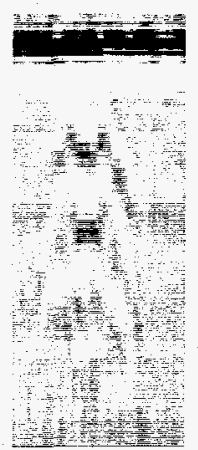

(a)

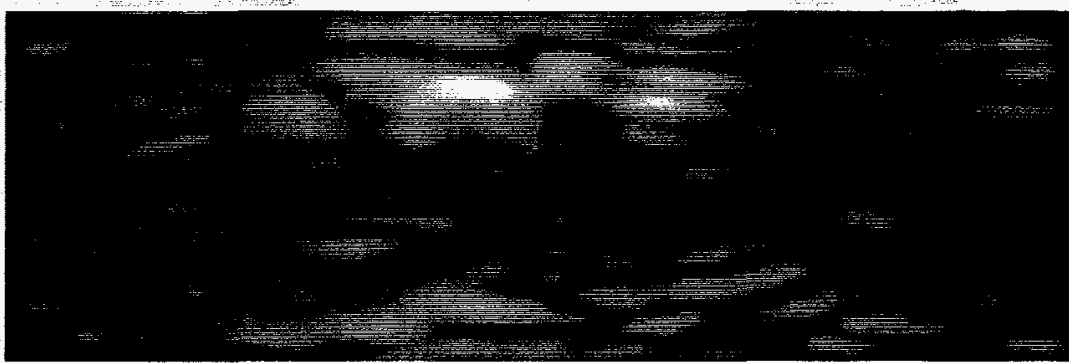

(b)

Figure 7. Raw 2-D radar data (a) and reconstructed 2-D image (b) of buried M-19 mine. 


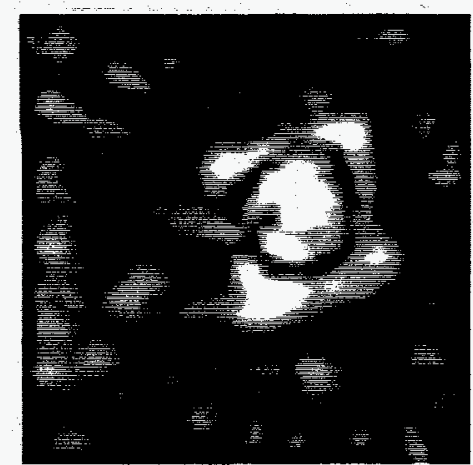

Depth $=4 \mathrm{~cm}$

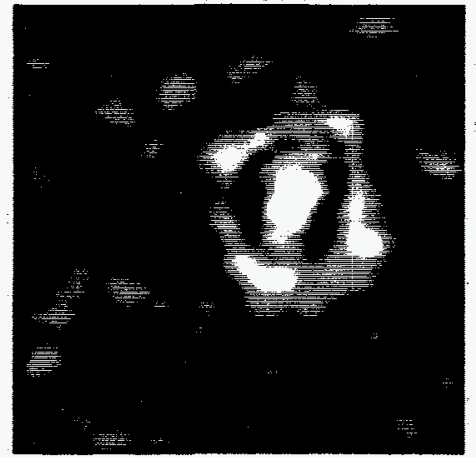

Depth $=5 \mathrm{~cm}$

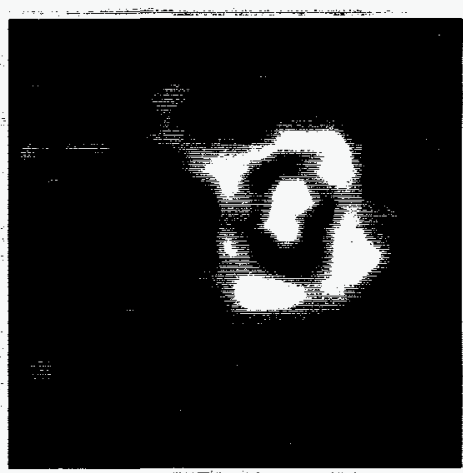

Depth $=6 \mathrm{~cm}$

Figure 8. Reconstructed image sequence for an M-19 plastic mine.

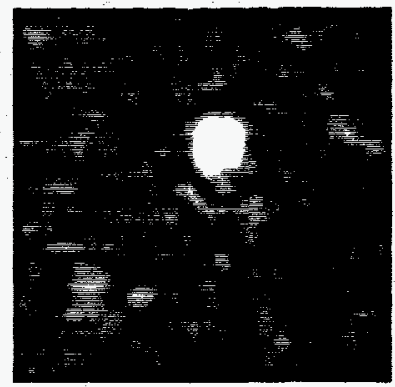

Depth $=5.5 \mathrm{~cm}$

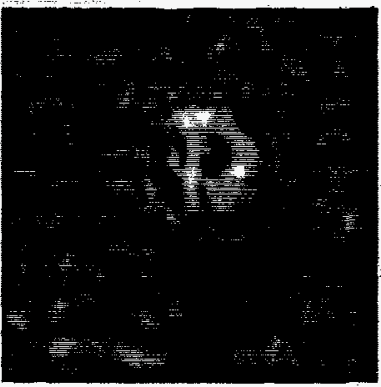

Depth $=7.0 \mathrm{~cm}$

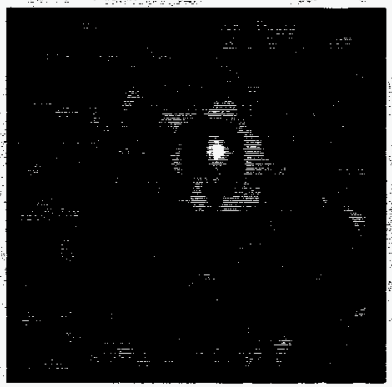

Depth $=7.5 \mathrm{~cm}$

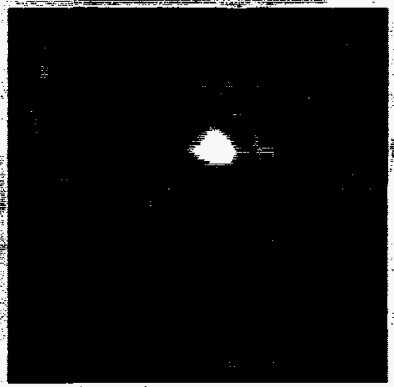

Depth $=9.0 \mathrm{~cm}$

Figure 9. Reconstructed image sequence for a VS-2.2 plastic mine.

3.5 The reconstruction of particular features of these mines shows that they have unique MIR signatures which could be used to differentiate them from natural objects like stones or rocks. In addition to various mines, we also imaged buried rocks of roughly the same dimensions as the mines. Figure 10 shows a 2-D reconstructed image of a roughly five-inch diameter rock buried at NTS. The two distinguishing factors for mines vs. rocks are the strength of reflected signal and the shape. While the rock in Figure 10 has a similar shape to a mine, its return value or intensity is different. In many other cases, shape can be used to distinguish mines from most irregularly-shaped rocks. Animal burrows in the ground were also imaged with similar results. Imaging is expected to provide low false alarm rates because of this ability to distinguish shape. However, this must be confirmed by further field studies and statistical analyses.

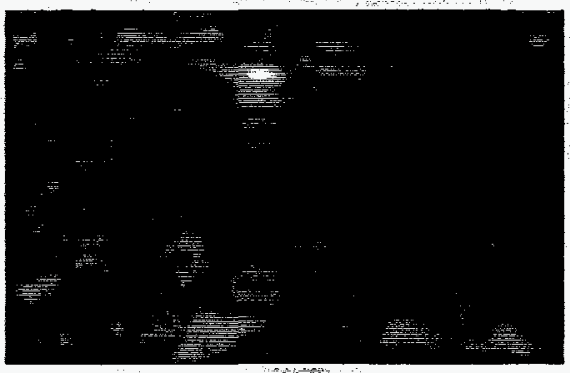

Figure 10. Reconstructed 2-D image of a buried rock. 
4.1 Vehicle-mounted and man-portable mine detection systems are needed for demining operations in both lowand high-clutter environments. MIR arrays and image reconstruction software can be readily integrated into viable multisensor systems that address both these needs. These systems can be used for detection and marking of individual mines, for personnel assessment and training, and for quality assurance.

4.2 For low-clutter environments, such as improved and unimproved roadways, a linear array of MIR modules configured in a "look-ahead" operating mode and mounted on the front of a remote-controlled ground platform can be used to detect anti-tank and anti-vehicular mines. This concept is shown in Figure 11. In this configuration, MIR modules are assembled into an array positioned so that its field-of-view covers an area of 2 to 4 square meters, a few meters in front of the ground platform. Radar data is telemetered via wireless, fiber optic, or hard-wired connection to the remote control point, where image processing and display hardware are located. Data acquired from the array is used to make 2-D images of the area in front of the vehicle. Images produced by a "look-ahead" system provide information about the position of buried objects in a single subsurface horizontal plane, much like the images in Figures 8 and 9. Images of this type can be reconstructed at very high rates (at or near real-time). In this system concept, it is assumed that target burial depth is constant; i.e., all targets of interest are on the surface or buried shallowly in the top layer of the roadway. Where surface clutter is minimal, such as on roadways, this is a reasonable assumption.

4.3 In higher clutter environments, like off-road areas, a linear array of MIR modules can be configured for "Iookdown" operation to detect anti-tank and anti-personnel mines. The array is mounted on a boom extending in front of the remote-controlled vehicle to provide an appropriate standoff distance between the vehicle and the area being surveyed. In this case, the MIR array is mounted so that its field-of-view covers an area of 2 to 4 square meters below the array. The array is scanned so that a 2-D aperture is synthesized. Again, radar data is telemetered via wireless, fiber optic, or hard-wired connection to the remote control point. Data acquired from the synthetic array is used to reconstruct 3-D images of the scanned area. This approach is useful in areas where vegetation and other surface obstacles are present, because target position and burial depth can be determined from the reconstructed image. Surface clutter sources can be identified and removed from the image, if they are determined by other vehicle mounted sensors not to be mines, so that the buried objects can be detected, classified, and marked.

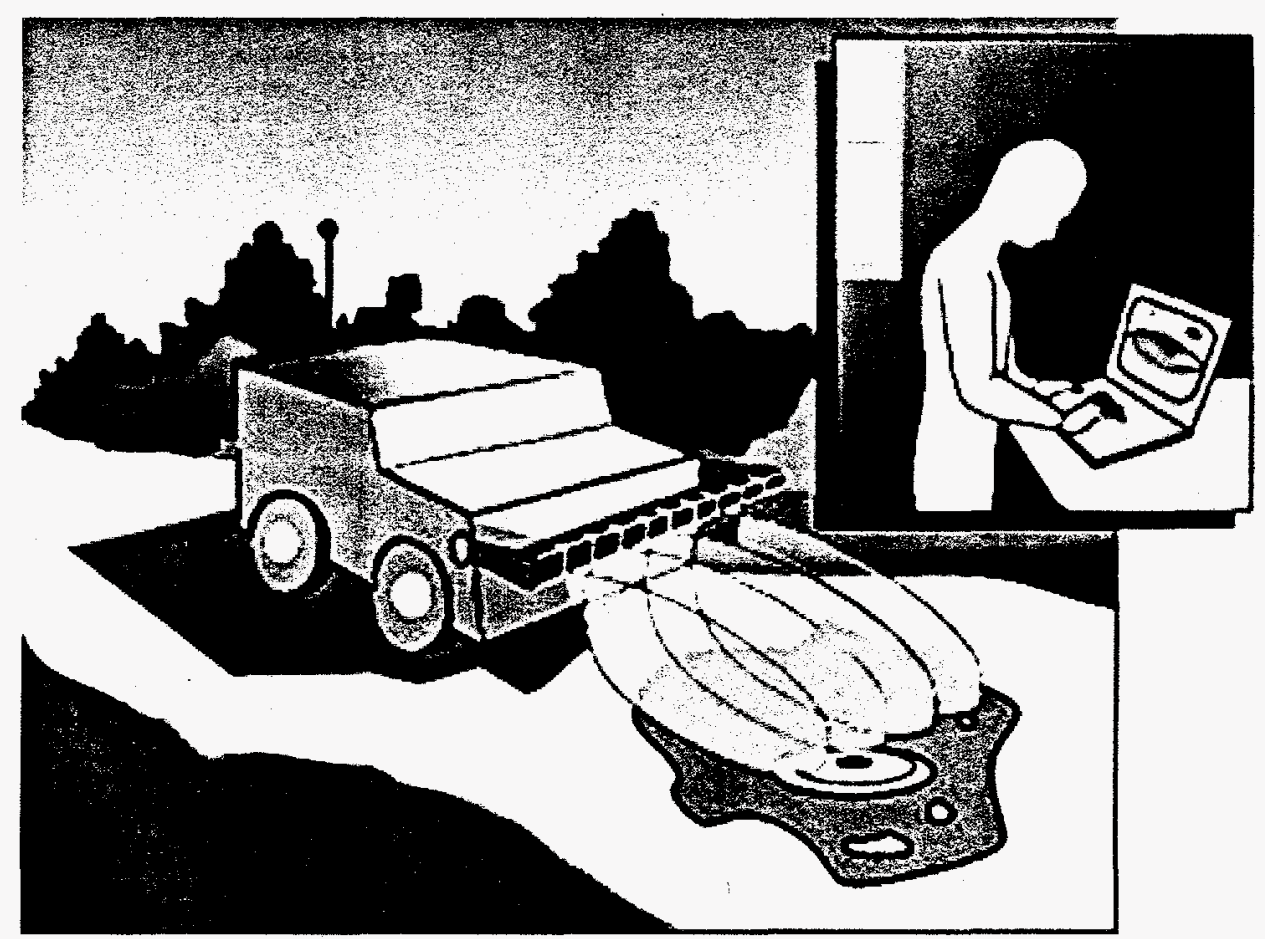

Figure 11. "Look-ahead" MIR-based mine detection concept. 
4.4 Man-portable mine detection systems may also be required in demining operations. In areas where terrain, foliage, and other obstacles preclude the use of remote-controlled detection systems, man-portable detection techniques may be the only methods available for detecting mines. Compact size, light weight, and low cost make MIR technology an excellent candidate for these systems. Even imaging arrays that operate in the "look-ahead" mode and quickly produce 2-D maps of areas searched by human operators are feasible with current technology.

4.5 Some modifications are required to adapt current MIR module technology for the system concepts described above. Several development activities, already underway at LLNL, address these needs. For example, ongoing design improvements in the MIR receiver will integrate a low noise, wideband amplifier into the front-end, reducing the noise figure to 4 to $5 \mathrm{~dB}$ and extending the dynamic range to $98 \mathrm{~dB}$. Impulse generator development work is also underway to integrate step recovery diodes into the transmitter output stage to increase bandwidth and peak power. These changes will permit MIR modules and arrays to operate at stand-off ranges required for vehicular and manportable mine detection systems used in demining operations. In addition, work is progressing on antenna and antenna lens designs to tailor antenna gain and radiation patterns for the needs of a variety of applications. Our preliminary analysis of requirements for MIR modules used for mine detection are summarized in Table 2.

Table 2. MIR module requirements for mine detection applications

\begin{tabular}{|c|c|c|c|c|c|c|}
\hline 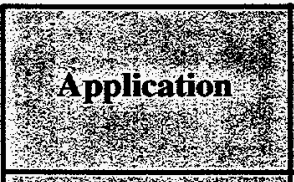 & 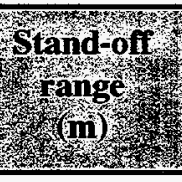 & 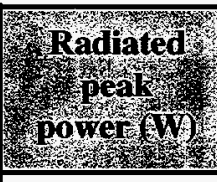 & 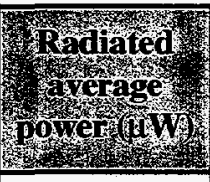 & 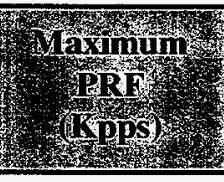 & 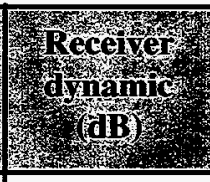 & 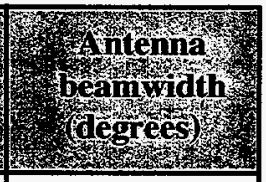 \\
\hline 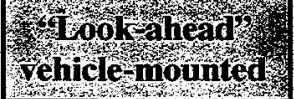 & 2 to 3 & 52.7 & 10 & 1.9 & 90 & 60 to 75 \\
\hline 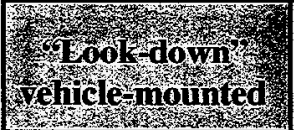 & 0.5 to 1 & 0.75 & 10 & 133 & 90 & 75 to 90 \\
\hline 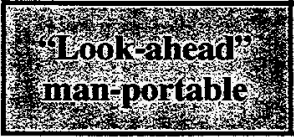 & 1 to 1.5 & 3.5 & 10 & 28 & 90 & 60 to 75 \\
\hline
\end{tabular}

\subsection{Summary}

5.1 MIR and radar imaging technology developed at LLNL have direct applicability to the demining problem. Our feasibility tests have shown that these technologies reliably detect and image both metallic and plastic mines, and that they offer the potential for classifying buried objects that could improve detection and decrease false alarm rates for multisensor mine detection systems. Imaging speed currently gives near-real-time results on a laptop computer, and is expected to improve with high-speed portable computers. Costs of such a system will be low, even with imaging capability, because hardware costs of the MIR are orders-of-magnitude below current radar technology. Ongoing development activities at LLNL are addressing design changes that are needed to further improve current MIR technology to satisfy mine detection requirements.

5.2 LLNL has extensive resources, facilities, and capabilities for developing radar imaging systems including microwave electronics, RF diagnostics, antennas, and imaging technology. Our existing facilities include laboratories dedicated to testing and evaluating MIR hardware, and computer and related facilities needed to support electromagnetic modeling, signal processing and image reconstruction development, and data visualization needs. In addition, the MIR program has access to other LLNL facilities, including antenna test ranges and anechoic chambers, supercomputers, and a variety of engineering workstations. The program is directly supported by personnel with extensive experience in analysis, design, development, and testing of radar systems, electromagnetics and antennas, EM modeling, simulation, signal processing and imaging algorithms.

5.3 Minefield clearing is an important international humanitarian problem. LLNL stands ready to apply its unique capabilities to this problem in partnership with companies or agencies in a position to field and market technical solutions. We plan to seek sponsorship and to continue to develop our expertise in this area. The MIR and 
imaging technologies will be non-exclusively licensed to companies who can demonstrate an ability to package and deliver demining equipment throughout the world. MIR technology has already been licensed for use in automotive and handtool applications, and licenses are pending to several firms for application to diverse fields including medicine, construction, and law enforcement.

\subsection{References}

[1] P.D. Sargis, F.D. Lee, E.S. Fulkerson, B.J. McKinley, W.D. Aimonetti, "Ground-Penetrating Radar for Buried Mine Detection," SPIE Vol. 2217, Aerial Surveillance Sensing, including Obscured and Underground Object Detection, 4-6 April 1994.

[2] J.E. Mast, E.M. Johansson, “Three-dimensional ground penetrating radar imaging using multi-frequency diffraction tomography," SPIE Vol. 2275, Advanced Microwave and Millimeter Wave Detectors, 25-26 July 1994. 


\section{Appendix A: Micropower Impulse Radar}

\section{A.1. MIR Motion Sensor Development}

The Micropower Impulse Radar (MIR) is a fundamentally different type of radar that was invented and patented by Lawrence Livermore National Laboratory (LLNL). It is a pulsed radar like other ultra-wideband radars, but it emits much shorter pulses than most and, because it is built out of a small number of common electronic components, it is compact and inexpensive. The genesis of these radars starts with LLNL's 100 -trillion watt Nova laser, the world's highest power laser system, which was developed for nuclear fusion research. The pulsed laser generates highspeed (sub-nanosecond) events that must be recorded. In answer to this need, special fast electronic circuitry was developed for driving a 33 gigasample-per-second transient digitizer that records such events. Thomas McEwan, the LLNL engineer who designed this high-speed data acquisition system, then had an important insight: these new circuit concepts could be augmented by additional electronics into extremely small, low-power radar systems.

Soon after McEwan's breakthrough in 1993, it became evident that such radars had the possibility of impacting an extremely wide range of applications. Since then, nearly 30 patents have been filed, hundreds of commercial applications have been identified and MIR technology has become LLNL's biggest technology transfer activity. Licenses with industry have been signed in the areas of automobile back-up systems and in hand-held tools for finding studs and other objects behind walls. Many other applications are under investigation including such diverse fields as fluid level sensing (electronic dipsticks), heart monitoring for medical applications, security systems, detection of breathing through walls or rubble (e.g., finding survivors of earthquakes), monitoring of infants for the possible prevention of Sudden Infant Death Syndrome (SIDS), underground and through-wall imaging, and many others.*

McEwan and his team continue to innovate and develop new ideas for the MIR technology including new electronics, antennas, signal processing, and imaging concepts. LLNL is following the dual paths of licensing the technology to qualified manufacturers and developing programs that use the technology in support of the laboratory mission.

One unique feature of the MIR is the pulse generation circuitry, which, while small and inexpensive, had never before been considered in radar applications. Each pulse is less than a billionth of a second and each MIR emits about two million of these pulses per second. Actual pulse repetition rates are coded with random noise to reduce the possibility of interference from other radars, while each is "tuned" to itself. The same pulse is used for transmitting as is used for sampling the received signal. Three direct advantages of the short pulse-width are as follows:

1. With pulses so short, the MIR operates across a wider band of frequencies than a conventional radar, giving high resolution and accuracy, but also making it less susceptible to interference from other radars.

2. Since current is only drawn during this short pulse time and the pulses are infrequent, there are extremely low power requirements. One type of MIR unit can operate for years on a single AA battery.

3. The microwaves emitted by the pulse are at exceedingly low, and therefore medically safe, levels (microwatts). Indeed, the MIR emits less than one-millionth the energy of a cellular telephone!

Probably the main unique feature of this radar is the cost. The current version uses off-the-shelf electronic components so that a standard MIR board can be assembled with less than $\$ 20$ of parts. Future development plans include reducing the MIR components to multi-chip modules or ASIC's as the demand increases and it becomes economically feasible.

As with conventional radars, the antenna configuration on the MIR determines much of its operating characteristics. Several antenna systems have been designed to match the ultra-wide frequency characteristics of the MIR sensor. For the standard MIR motion sensor with a center frequency of about $2 \mathrm{GHz}$, we use a small 1.5-inch antenna. However, the MIR is also flexible enough so that it can operate at a relatively lower center frequency, using larger antenna systems, giving it longer range and better capability for penetrating water, ice, and mud.

What makes the MIR so useful for security applications such as border surveillance is its range-gating capability. Imagine that each radio pulse is a large wave traveling across a lake. The wave bounces off an island and comes

* See the March 1995 issue of Popular Science where McEwan and the MIR are featured in the cover article. 
back to you. The amount of time it takes the wave to return depends on the distance to the island. By setting the radar's "gate", or echo acceptance range, to open only at the right time to receive echoes from a certain distance, it can ignore all other echoes. Range gating can therefore be used to set up an invisible security bubble around the radar. In a burglar alarm, for instance, the range might be set at 20 feet. The radar then detects only objects that modulate the reflected signal at this distance. It detects motion by repeatedly checking the echo pattern to see whether it changes over time; a change means the bubble has been penetrated by a moving object. This eliminates triggering on stationary background objects or "clutter".

The current MIR motion sensor can be fully concealed behind walls or inside drawers while detecting intruders at ranges up to 20 feet. Its sharply-bounded detection range is easily adjusted for any situation. In addition, the MIR can project a set or sweep of range shells to generate a filled volume of sensitivity. It does not respond to objects outside the current range gate, and it attempts to avoid false triggering on near objects such as insects. However, the range-gating mechanism may be susceptible to triggering on large-object movement in the near-field (inside the range bubble) because radar phenomena like multiple scattering will modulate the range-gate return.

Averaging of many thousands of pulses is done on the MIR to reduce the effects of noise and to increase sensitivity. A single received pulse in the nanosecond time scale may be contaminated with various forms of outside interference, but if the returns of many pulses at the same range gate are combined, the result is much more representative of the actual return. The number of averages per range gate is adjustable (nominally it is about 10,000 samples) and is one of the tradeoffs in the MIR design.

The exact pulse emission and detection times are randomized for three reasons. First, continuous wave (CW) interference, such as from radio and TV station harmonics, may cause beat frequencies with the received echoes that can trigger false alarms. When the 10,000 samples of MIR return echoes are averaged at randomly-dithered times, random samples of $\mathrm{CW}$ interference are effectively averaged to zero. Second, random operation also means that multiple MIR units can be collocated without interfering with each other. Channel allocations are not needed and a nearly unlimited number of sensors can be in the same vicinity even though they occupy the same wideband spectrum. Third, randomizing also spreads the sensor's emission spectrum to the point that it resembles random thermal noise, making it difficult to distinguish from background noise. That is, this randomizing makes the MIR very stealthy.

A primary application for the MIR motion sensor is home intrusion detection. As an example of its use, the MIR sensor can be mounted on a ceiling, perhaps disguised as a smoke alarm, and its range gate, or detection shell, can be set to six feet to intercept intruders below while not extending down to the floor where pets may trigger it. Other concealment possibilities spring to mind: in a cookie jar, in a hollowed-out book on a shelf, behind a wall-painting near the front door, or in a shoe box in a closet. In a typical installation, the MIR would use a radio transmitter to alert a central alarm system.

With the freedom provided by battery operation and the ability to operate through barriers, MIR can be installed in seconds. Installation typically involves no more than setting the desired operating range and perhaps a radio transmitter code, and then hiding the unit.

The MIR sensor provides an unprecedented combination of features:

- Ability to "see" through walls, allowing concealment

- Sharply defined maximum operating range, reducing false alarms

- Several year battery life, simplifying installation

- Collocation of multiple units without interference, simplifying installation

- Randomized spread-spectrum emissions, making the sensor difficult to detect

- Very low cost, using entirely off-the-shelf components

- Single silicon chip integration is possible, for low cost and size. 
General specifications:

Antenna pattern (H-plane)

Center frequency

Emission bandwidth

Average emission power

Duty cycle

PRF (average)

PRF coding

Receiver noise floor

Receiver gate width

Range delay

Range delay jitter

Range delay stability

Detection range

Motion passband

Analog output

Receiver gain

Power

Size

Semiconductors $360^{\circ}$ with a dipole antenna,

$160^{\circ}$ with a cavity-backed monopole

1.95 or $6.5 \mathrm{GHz}+/-10 \%$

$500 \mathrm{MHz} @ 1.95 \mathrm{GHz}$ center

$\sim 1 \mu \mathrm{W}$ (measured)

$<1 \%$

$2 \mathrm{MHz}+/-20 \%$

Gaussian noise, or low coherence

swept FM, or pseudo-noise

$<1 \mu \mathrm{V}$ rms

$250 \mathrm{ps}$ for $1.95 \mathrm{GHz}$ system

$\mathrm{RC}$ analog, pot/DAC controllable

$<1 \mathrm{ps}$ rms

RC component limited over temperature

(drift in range delay expands/shrinks shell)

adjustable from 2" inches to $>20$ "

$0.3-10 \mathrm{~Hz}$, Doppler-like signature

$\sim 0.1-2 \mathrm{~V}$ peak on motion sensing,

hand motion at 6' gives $\sim 300 \mathrm{mV}$ peak

$70 \mathrm{~dB}$

$5 \mathrm{~V} @ 8 \mathrm{~mA}$, normal power mode

$2.5 \mathrm{~V} @ 20 \mu \mathrm{A}$, long battery life version

1.5"square SMT PCB with 1.5 " long wire

dipole elements

$74 \mathrm{ACO0}$ CMOS (1ea),

bipolar or CMOS op amps (2 ea, quads),

Si-bipolar RF transistor @ $>4 \mathrm{GHz} f_{\mathrm{t}}$ (2ea.),

Schottky diodes, $\mathrm{C}_{\mathrm{j}}(0)<1 \mathrm{pF}$ (2ea) 


\section{A.2. Microradar rangefinder}

The radar rangefinder is a compact, low-cost ultra-wideband radar with a swept range gate. The device generates an equivalent-time A-scan (echo amplitude vs. range, similar to a WW-II radar) with a typical range sweep of 4" to 10' and an incremental range resolution, as limited by noise, of .01".

Uses include replacement of ultrasound rangefinders for fluid level sensing (a dipstick without the stick), vehicle height sensing, and robotics control. When positioned over a highway lane, it can collect vehicle count, vehicle profile, and approximate speed data for traffic control. Some versions transmit $\mathrm{RF}$ packets rather than a short impulse, and can provide swept-range quadrature Doppler information.

A major application for this microradar is imaging (see below). It operates in spectral regions that readily penetrate walls, control panels, and to an acceptable extent, concrete and human tissue.

The basic antennas have a very broad beamwidth and corresponding low gain. They are suitable for synthetic beam imaging where broad illumination is desirable. Narrower beamwidths and higher gain can be obtained on a broadband basis with horns, reflectors or dielectric lenses.

\section{General specifications:}

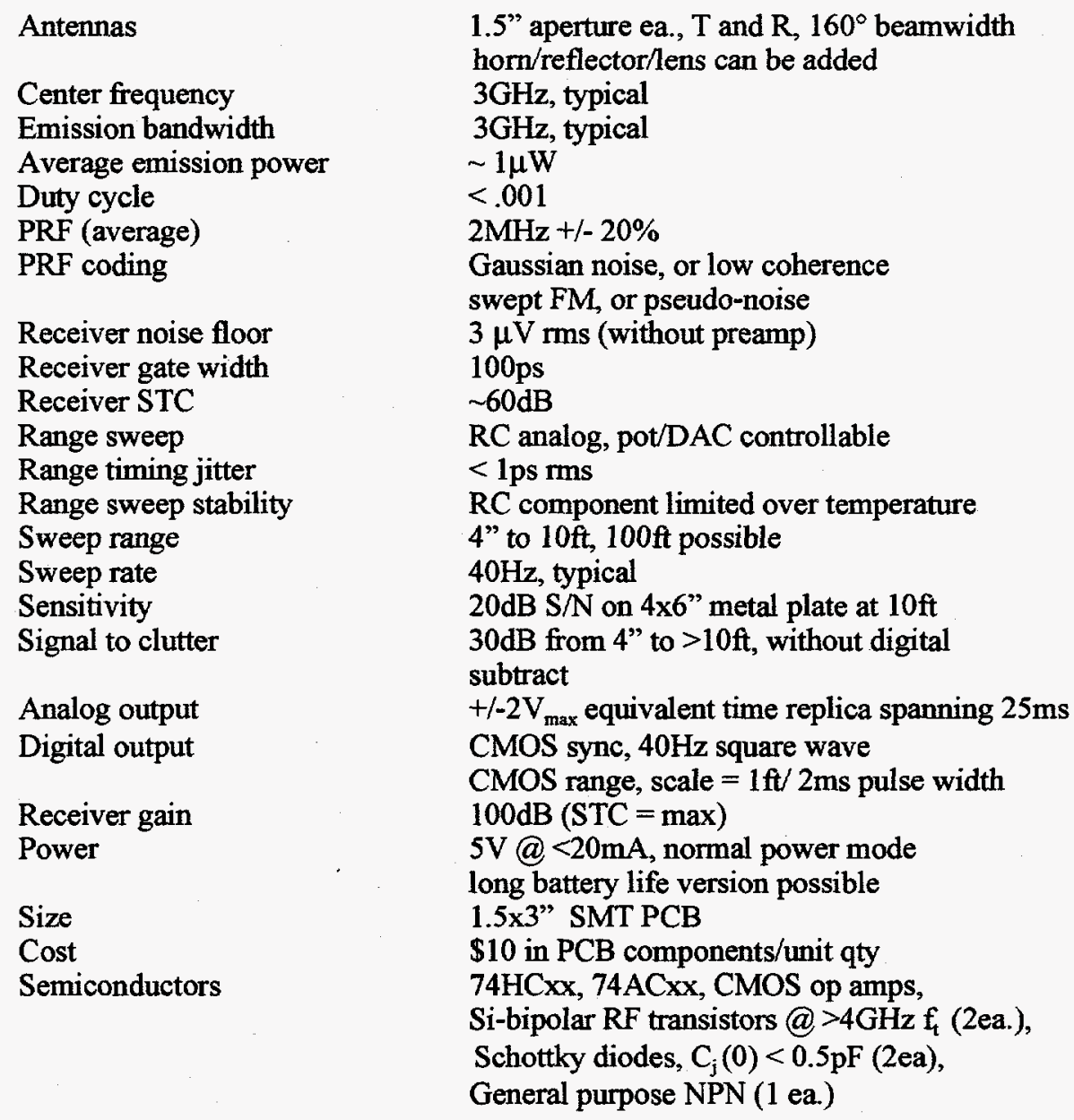

The STC (sensitivity-time control) increases voltage gain with $\mathrm{R}^{2}$. In addition, a peak-sensing AGC (automatic gain control) can be provided to maintain a constant receive pulse amplitude independent of range or target characteristics. For fluid level sensing, return pulse amplitude is held precisely constant 
and independent of fluid dielectric constant, so accuracy remains unchanged for aqueous and petroleum based liquids. Scale factor linearity is presently limited to $1 \%$ of full scale. Versions with long-term scale factor errors of $<.01 \%$ will be available in mid- 1995 .

\section{A.3. Micropower Impulse Radar Imager}

The MIR imager consists of small, low power, broadband radars (such as the microradar rangefinder above), that are being developed for a wide range of imaging applications. These radars are coupled to antenna arrays and a portable computer to form a complete radar imaging system. Software is used to reconstruct $2 \mathrm{D}$ and $3 \mathrm{D}$ views of the scene.

Due to their low-cost and small size, numerous MIR sensors can be assembled into arrays for syntheticand real-aperture image formation in 2D and 3D. Radar return signals are digitized and stored in a portable lap-top computer. Reconstruction of cross-sectional images from B-scan or waterfall type data is performed by diffraction tomography software on the lap-top. Images of the scene are displayed directly on the screen within 10 seconds (in 2D).

We have developed one array of 32 sensors for test and evaluation, and others are being designed. The imaging system detect differences in the dielectric constant of the materials under inspection-larger differences cause larger radar return signals. Resulting images, then, have high intensities at interfaces between materials of differing electrical properties. Effective resolution of the current system is about $1-2 \mathrm{~cm}$, depending on the medium and depth. The current array uses monostatic imaging, but future versions will be capable of multistatic operation.

General Specifications: Same as the microradar rangefinder above; variable depth (range) resolution, wideband pulser for fine cross-range resolution, briefcase-sized for portability, various antennas from $100 \mathrm{MHz}$ up to $5 \mathrm{GHz}, 2 \mathrm{D}$ imaging in less than 10 seconds, long battery life, exceedingly low emissions, and low cost.

\section{Applications:}

- Ground-penetrating imaging for mines and buried ordnance

- Through-wall detection of people

- Nondestructive evaluation of civil structures

- Inspection of road beds and bridges

- Location of rebar and conduit in concrete

- Moisture content locator in wood, soil or concrete 